\title{
Klinische Studien - Weg nach vorne oder Schritte zurück?
}

\author{
Sonja Kickmaier, Heinz Kölbl
}

Definition. „Die klinische Prüfung von Arzneimitteln ist eine am Menschen durchgeführte Untersuchung, die dazu bestimmt ist, klinische oder pharmakologische Wirkungen von Arzneimitteln zu erforschen oder nachzuweisen oder $\mathrm{Ne}$ benwirkungen festzustellen $(\S 4$, Absatz 23 Arzneimittelgesetz, AMG). Im Rahmen der Entwicklung eines neuen Therapieansatzes stellen die klinischen Studien den letzten Schritt in einer langen Entwicklung zum fertigen Medikament dar. Sie sind ein wichtiger Bestandteil der universitären Pharmaforschung, bei der es im Wesentlichen um die Verträglichkeit und/ oder Wirksamkeit von Medikamenten oder operativer Verfahren geht. Abhängig von der Fragestellung können viele Arten von Studien zum Einsatz kommen, etwa zur Evaluation von neuen chirurgischen oder radiologischen Therapieansätzen oder Medizinprodukten, deren Design im Medizinproduktegesetz festgeschrieben ist. Abhängig vom Stadium der Entwicklung der Intervention können kleinere
Pilotstudien oder große internationale Multicenterstudien durchgeführt werden.“ (Abb. 1) [10].

\section{Nachfrage nach „neuen“ Medikamenten}

Zum Weltkrebstag haben Experten prognostiziert, die „globale Krebsepidemie ist auf dem Vormarsch“. Nach Angaben der „Union international contre le cancer“ (UICC) erhalten derzeit jedes Jahr mehr als 12 Millionen Menschen weltweit die Diagnose Krebs, 7,6 Millionen sterben an einer Krebserkrankung. Die Tendenz ist steigend. Experten schätzen, dass im Jahr 2030 jährlich weltweit rund 26 Millionen Menschen an Krebs erkranken und 17 Millionen Menschen daran sterben werden $[6,7]$.

Umso bedeutungsvoller ist es, zukünftig dafür zu sorgen, dass die Entwicklung sog. „neuer“ Medikamente, welche als wesentlich spezifischer und nebenwirkungsärmer gelten, voranschreitet und die $\mathrm{Zu}$ lassung der Neuentwicklungen rasch vor sich gehen kann (Abb. 2).

\section{Regelungen für Neuzulassungen}

Die gesetzlichen Bestimmungen zur Neuzulassung dieser hochwirksamen Medikamente sind streng geregelt. So muss jedes Therapeutikum mehrere unterschiedliche Studienphasen durchlaufen, bevor es generell als Therapie zugelassen werden kann (Tab. 1).

Studienziele. Klinische Prüfungen dürfen nur durchgeführt werden, wenn sie erwartungsgemäß die folgenden Zielsetzungen des § 2a Abs. 1 des AMG erfüllen:

- Wirkungen von Prüfpräparaten zu erforschen oder nachzuweisen (Abb. 3)

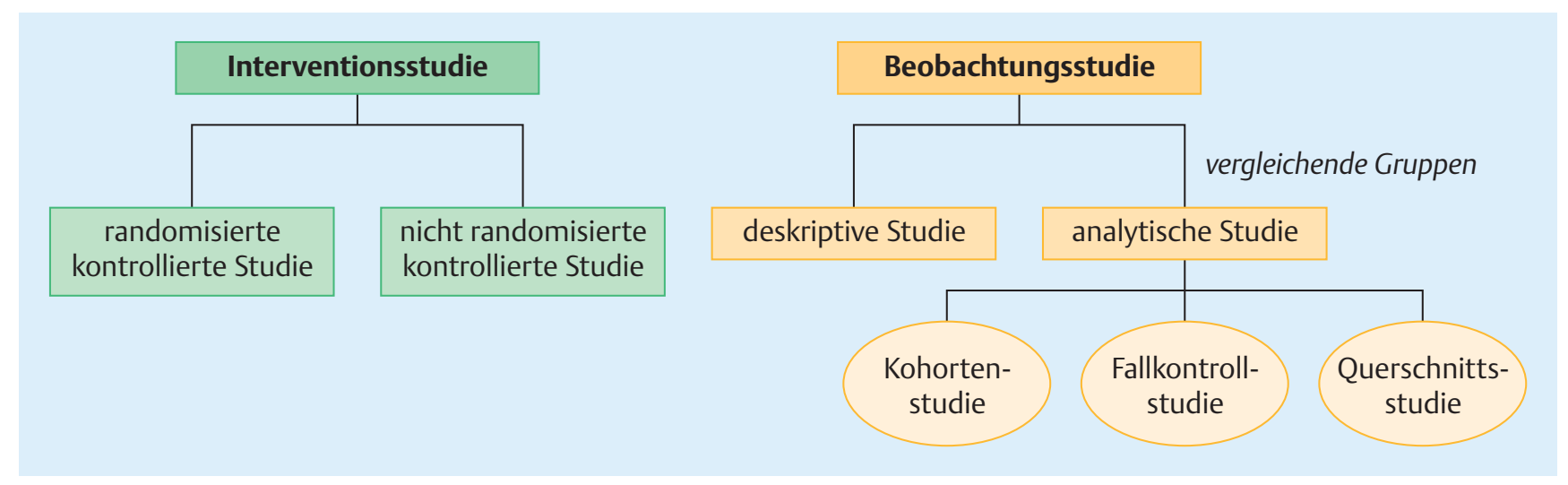

Abb. 1 Unterschiedliche Varianten von Studien. 


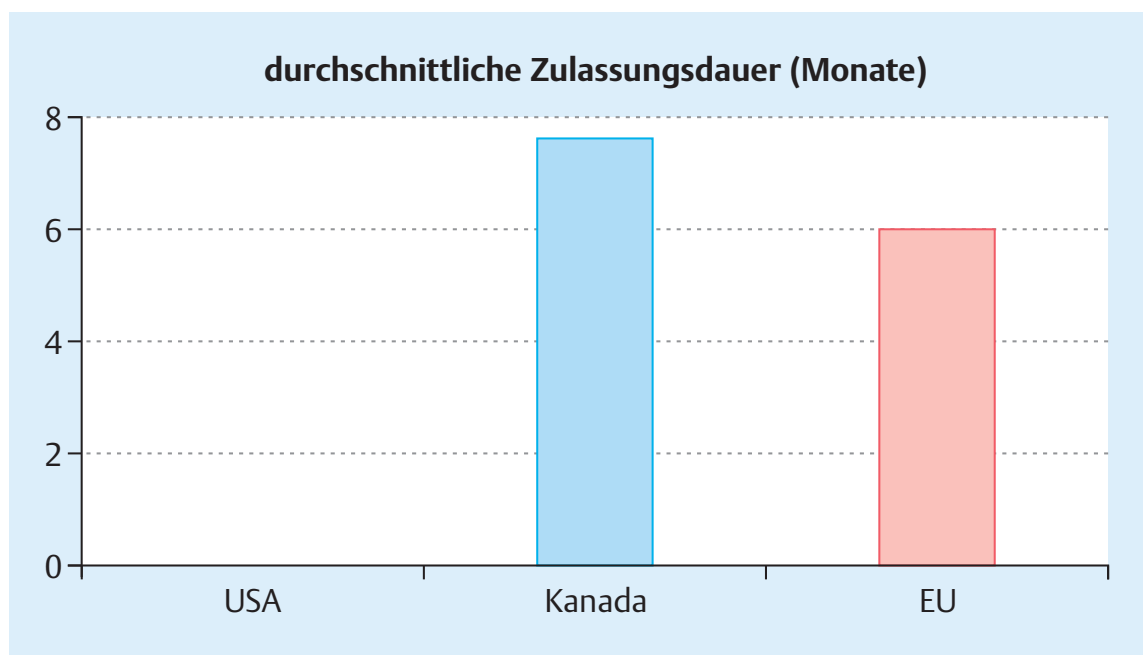

Abb. 2 Durchschnittliche Zulassungsdauer nach Zulassung durch die FDA von Medikamenten in den USA, Kanada und der EU im Vergleich (Quelle: [8]).

\section{Tabelle 1}

Die verschiedenen Studienphasen auf dem Weg zur Neuzulassung eines Medikaments.

\begin{tabular}{|c|c|c|c|}
\hline Phase & Zeitraum & Anzahl Testpersonen & Studien-/Phasenziel \\
\hline 0 - Micro Dosing & einige Wochen & $10-15$ & Pharmacodynamics \\
\hline I - Safety & Wochen bis Monate & $20-80$ & $\begin{array}{l}\text { Safety, Digestibility, } \\
\text { Proof of Concept lla }\end{array}$ \\
\hline II - Dose Finding & Wochen bis Monate & $50-200$ & Dose Finding Ilb \\
\hline $\begin{array}{l}\text { III - Verification } \\
\text { of Concept }\end{array}$ & Monate bis Jahre & $200-10000$ & $\begin{array}{l}\text { Significant Verification } \\
\text { of Concept }\end{array}$ \\
\hline IV - Marketing & Jahre & $1000-1000000$ & Marketing, Safety \\
\hline
\end{tabular}

- Nebenwirkungen von Prüfpräparaten festzustellen

- die Resorption, die Verteilung, den Stoffwechsel und die Ausscheidung von Prüfpräparaten zu untersuchen

- Angaben über relevante physikalische und chemische Daten dokumentiert sind und aussagefähige Ergebnisse nicht klinischer Prüfungen vorliegen

Prüfarzt. Prüfer darf nur ein Arzt oder Zahnarzt sein, der zur selbstständigen Ausübung seines Berufes berechtigt ist und folgende Kriterien erfüllt:

- über entsprechende Kenntnisse und Erfahrungen auf dem Gebiet der klinischen Prüfung von Arzneimitteln verfügt

- über entsprechende Kenntnisse und Erfahrungen auf dem vorgesehenen Indikationsgebiet und über biometrische Kenntnisse verfügt

Antragstellung. Vor Beginn einer klinischen Prüfung hat der Sponsor (Pharma) beim Bundesamt für Sicherheit im Gesundheitswesen einen ordnungsgemäßen Genehmigungsantrag zu stellen und dabei alle für die Beurteilung der klinischen Prüfung erforderlichen Unterlagen vorzulegen und die Stellungnahme einer Ethikkommission einzuholen.

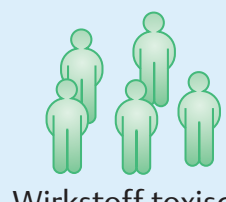

Wirkstoff toxisch, aber wirksam

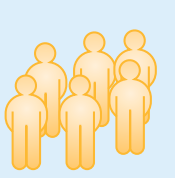

Wirkstoff nicht toxisch, aber auch nicht wirksam
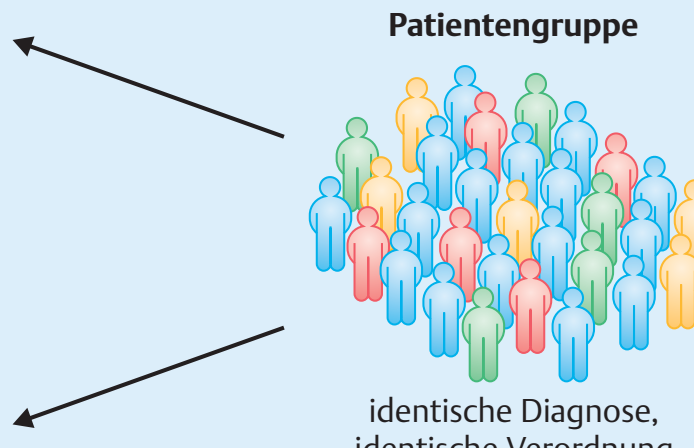

identische Diagnose, identische Verordnung

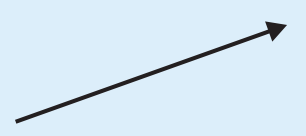

Wirkstoff toxisch, aber nicht wirksam
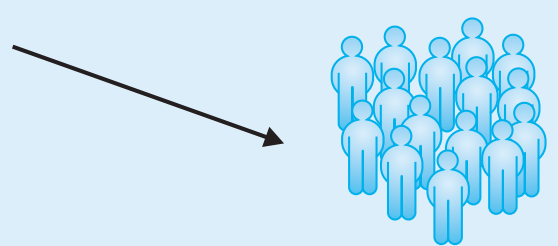

Wirkstoff nicht toxisch, aber wirksam

Abb. 3 Spezifischer Wirkungsvorteil. 


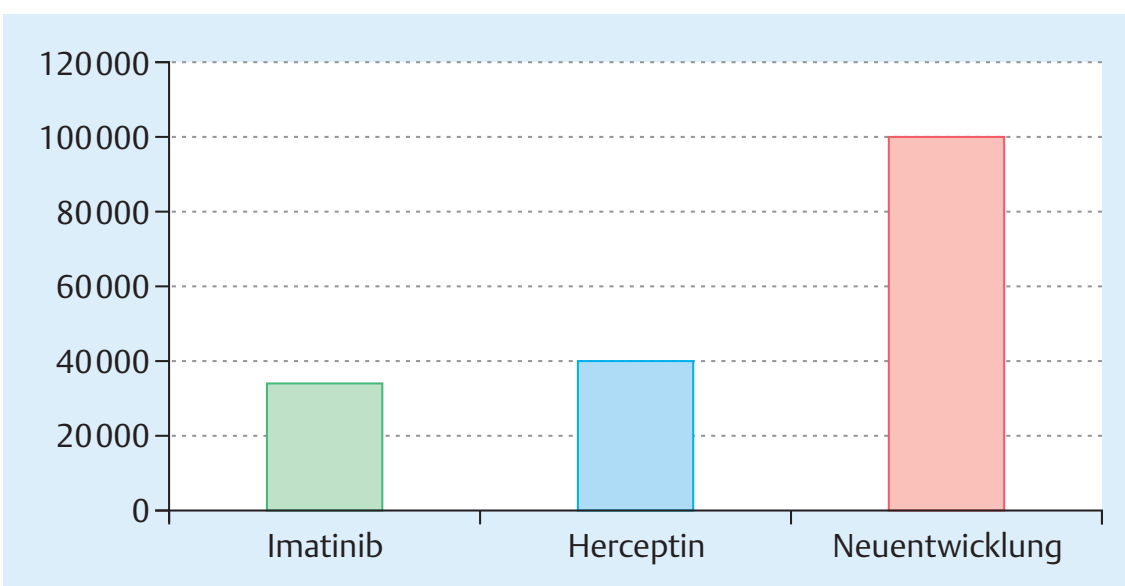

Abb. 4 Therapiekosten pro Jahr und Patient in Euro (Quelle: [7]).

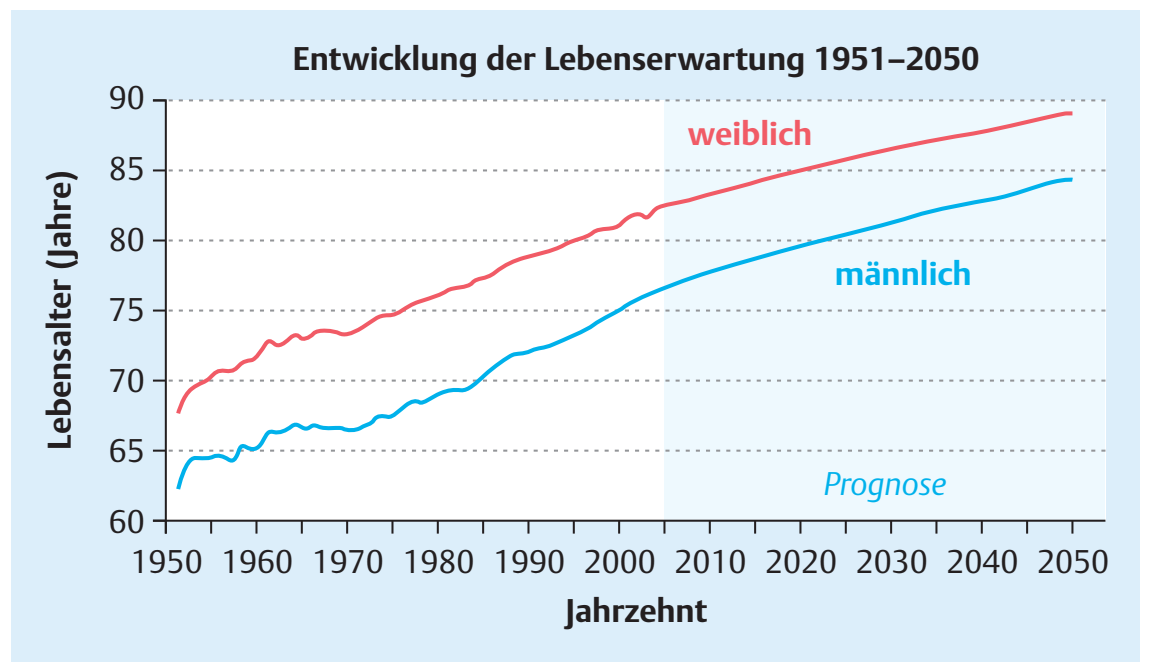

Abb. 5 Zunehmende Nachfrage durch steigende Lebenserwartung (3 Monate/Jahr seit 1954) (Quelle: [7]).

\section{Good Clinical Practice - GCP}

Klinische Prüfungen werden nach GCP Good Clinical Practice - durchgeführt. GCP ist ein internationaler ethischer und wissenschaftlicher Standard für Planung, Durchführung, Dokumentation und Berichterstattung von klinischen Prüfungen am Menschen. Die Einhaltung dieses Standards schafft öffentliches Vertrauen, dass die Rechte, die Sicherheit und das Wohl der Prüfungsteilnehmer gemäß der Deklaration von Helsinki geschützt werden und die bei der klinischen Prüfung erhobenen Daten glaubwürdig sind. Die
Maß an Überregulierung ist ebenso notwendig wie umfangreiche Dokumente, die Art und Ablauf der Verlaufskontrollen, Blutentnahmen, Verwendung von Gewebeproben, Biologie der Erkrankung, Vertraulichkeit und Patientenrechte strukturieren, und um die Beziehungen zwischen privaten/öffentlichen Partnern und der Industrie neutral zu halten.

\section{Studien im Wandel}

\section{Personalisierte Medizin in der Onkologie}

Ergänzend zur Chemotherapie kommt mehr und mehr die personalisierte Medizin zum Einsatz. Da als Auslöser der Erkrankung zahlreiche, über das gesamte Genom verteilte verschiedene Mutationen festgestellt wurden, gewinnt die Antikörpertherapie zusätzlich noch an Bedeutung [4].

Da eine Therapie und die vorgehende Diagnostik solcher Gendefekte das Gesundheitsbudget enorm belasten, sind diese derzeit fast nur im Rahmen von Studien anwendbar.

Die Therapiekosten einer solchen Neuentwicklung belaufen sich auf etwa 100000 Euro pro Jahr und Patient. Die Kosten dafür sind von den Sozialversicherern zu tragen (Abb. 4).

Zielsetzung dieser ICH-GCP-Leitlinie ist es, für die Europäische Union (EU), Japan und die Vereinigten Staaten von Amerika (USA) einen einheitlichen Standard zu schaffen, der die gegenseitige Anerkennung klinischer Daten durch die Zulassungsbehörden in den jeweiligen Zuständigkeitsbereichen fördert.

Kooperative Zusammenarbeit. Die strengen Zulassungskriterien neu entwickelter Wirkstoffe erfordern nicht nur speziell ausgebildetes Personal, sondern auch eine enge Kooperation zwischen verschiedenen Teilbereichen der Kliniken, Pharmafirmen und Behörden auf inhaltlicher und politischer Ebene. Ein wohl dosiertes
Die eigentliche Revolution in der personalisierten Krebstherapie ist für die Speerspitze der onkologischen Forschung zwingend - nämlich die Etablierung einer neuen Studiengeneration, mit der es möglich ist, die Erfolge zielgerichteter Therapien bei mehr Patienten in kürzester Zeit umzusetzen (Abb. 5 u. 6).

Studien sind ein wichtiger Baustein in der Gesundheitsversorgung und erhöhen die Effektivität, die Sicherheit und Therapietreue bei lebenswichtigen Krebstherapien und bedeuten Fortschritt in der Forschung. 


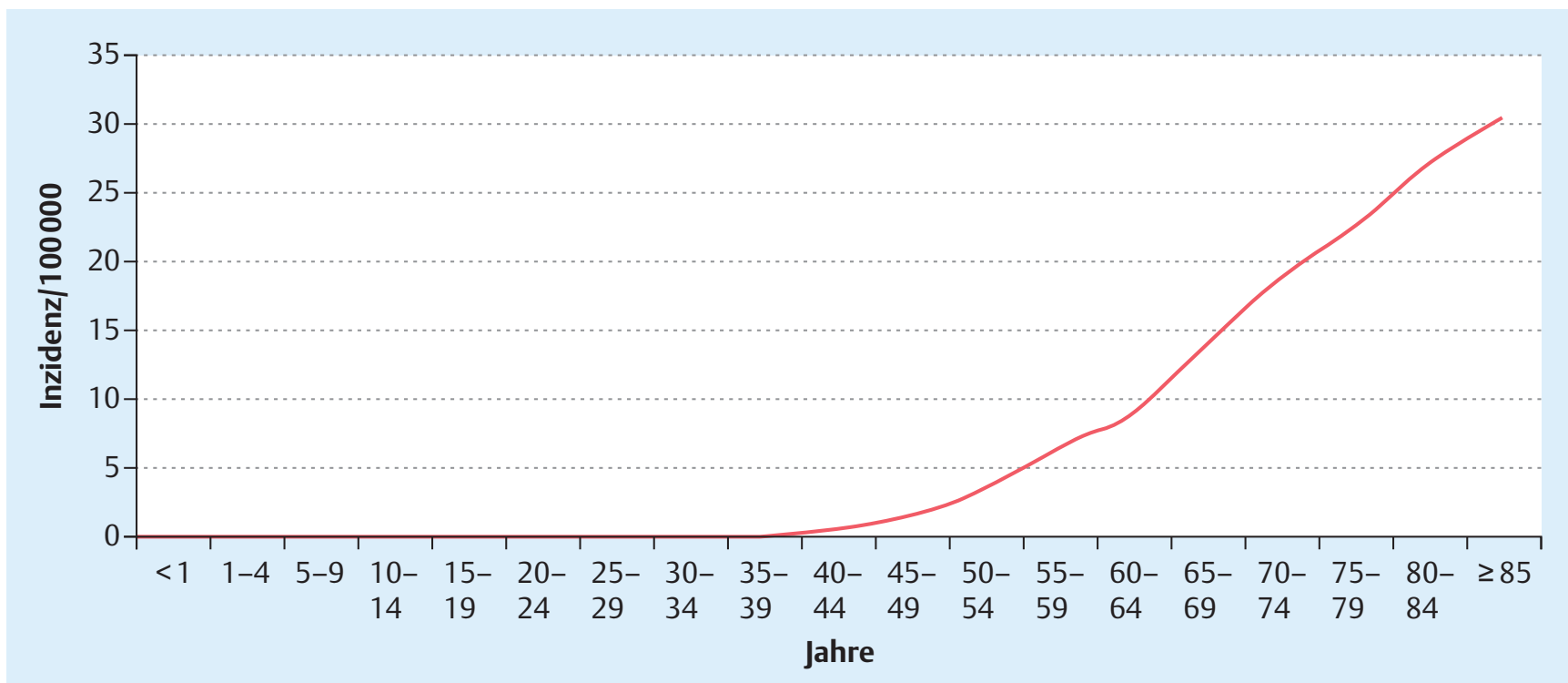

Abb. 6 Altersspezifische Inzidenz der CLL (USA, 1997-2001) (Quelle: [6]).

\section{Ethische Überlegungen}

Die dominierenden Diskussionen über die Kostenexplosionen im Gesundheitswesen führen zu der Annahme, dass hinter dem System der klinischen Studien finanzielles Interesse steht, das nicht dem Patienten dient, sondern der Bilanz des Auftraggebers. Dazu muss man sagen, dass wir ethische Standards haben, welche im Vergleich zu totalitären Ländern ungleich höher sind. Schwellenländer möchten immer mehr Teil dieser international klinischen Forschung sein, doch muss in den betreffenden Ländern erst eine transparente Struktur aufgebaut werden. Derzeit liegt dort ein Machtgefälle vor, da sich die Patienten meist nicht aus freien Stücken zur Studienteilnahme entscheiden können, sondern aufgrund sozialer Mängel keinen Zugang zu medizinischer Versorgung haben.

Es ist aber tatsächlich so, dass sich die Qualität einfach leichter kontrollieren lässt, wenn man „näher“ an der Kontrolle dran ist und die Studien strengen Standards entsprechen (Abb. 7).

\section{Strukturelle Anforderungen}

Die neuen zielgerichteten Therapien stellen auch an uns neue Anforderungen, z.B. hinsichtlich des Randomisierungsverfahrens (Zuteilung zu den Gruppen Standard + Verum oder Standard + Placebo nach vorheriger genauer Prüfung der Ein- und Ausschlusskriterien des Studienprotokolls mithilfe modernster Computerprogramme).

Bei Überlegungen bezüglich des therapeutischen Vorgehens sollten die wissenschaftlichen Erhebungen dabei auch zu einem Wandel in der öffentlichen Darstellung führen, da etablierte Behandlungen auf Basis verschiedener „Levels of Evidence" gründen, wobei diese im Wesentlichen durch wissenschaftliche Studien generiert werden.

- Starker Wettbewerb, Sicherung europäischer Wettbewerbsfähigkeit bei gleichzeitiger Wachstumsförderung in den USA, Asien und Lateinamerika

- Zugang zu Medikamenten für alle: eine globale Verpflichtung zur Solidarität, zu der wir unseren Beitrag leisten müssen
Im Vergleich zum Stand vor 10 Jahren sind Studien heute aufwendiger, qualitativ hochwertiger und erheblich teurer. Es braucht in Zukunft sicher mehr funktionierende Strukturen und enge Kooperationen zwischen Kliniken, Pharmafirmen, Pflegern sowie wohl auch einen Dialog zwischen Behörden auf inhaltlicher und politischer Ebene, um die Durchführung klinischer Prüfungen zu gewährleisten und Tendenzen wesentlicher Faktoren - positiv oder negativ - zu erkennen.

\section{Finanzierung}

Folgende Problemstellungen sollten ein Umdenken hin zu leistungsorientierter Finanzierung bewirken:

- knappe Hochschuletats

- abnehmende landesweite Förderung

- Compliance-Richtlinien: Pharmakonzerne und Universitäten

- Imbalance der Interessen: Industrie > Universitäten

- „Forschungslöcher“, z. B. operative Medizin

- Erfolgsrate Einreichungen Klinische Forschung (KLIF) 10\%

- KLIF mit 3 Mio Euro/Jahr limitiert 


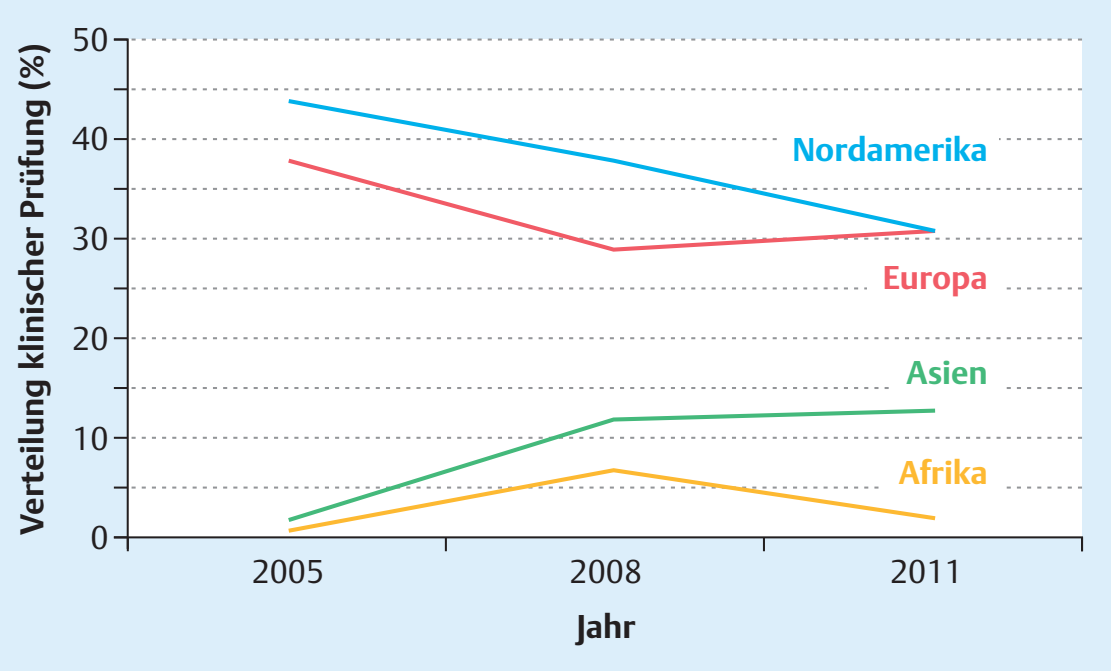

Abb. 7 Internationale Verteilung klinischer Prüfung.

Dennoch verlangen viele Studiensettings das Know-how und die Ausstattung großer Zentren, wenn z. B. in Phase-I-/IIStudien (Dosisfindungsstudien) Nebenwirkungen noch zu wenig bekannt sind und interdisziplinäre Betreuung vorhanden sein muss (s. Tab. 1).

Trotzdem sollte man das Zentren-Modell nicht dahingehend missverstehen, dass Patienten primär an einem Zentrum behandelt und betreut werden sollten, sondern das Credo lautet: Die Expertise soll reisen, nicht der Patient.

\section{Bereitschaft zur Studienteilnahme}

Wir leben heute in einer Epoche, die zum Motto hat: noch schneller, noch höher, noch stärker, noch extremer.

Unsere Patienten versetzt die Diagnose eines Tumors oder eines Rezidivs verständlicherweise in eine emotionale Achterbahnfahrt. Mitten in der Talfahrt lebenswichtige Entscheidungen zu treffen, ist schwierig genug, dennoch stellt sich ihnen gerade zu diesem Zeitpunkt auch die Frage der Teilnahme an einer klinischen Studie. Wichtig ist es uns dabei, die Patienten in Therapieentscheidungen mit einzubeziehen und damit die Compliance zu steigern. Die Erfahrung der letzten pie zugrunde liegen sollte.
Nutzenbewertung. Im Gegensatz zu Ländern wie den Niederlanden, England und Schweden führt man in Deutschland und Österreich sog. Nutzenbewertungen durch - aber keine Kosten-Nutzen-Bewertung. Diese Tatsache stößt insofern eine wichtige gesellschaftliche Diskussion an, als dass es dadurch nur bedingt zu einer neutralen Gestaltung der Medikamentenkosten kommt.

PFS als Parameter. Um aber der raschen Entwicklung neuer Medikamente besser Rechnung zu tragen, ist man dazu übergegangen, die Zeit bis zum Widerauftreten oder bis zum neuerlichen Wachstum des Tumors als Parameter heranzuziehen (Progression-free Survival; PFS).

Jahre zeigt, dass wir durch die intensive Aufklärung vor Beginn einer Studie sowie durch die ständige Betreuung (Erhebung der Lebensqualität und der subjektiv und objektiv empfundenen Beschwerden) während der Untersuchungsphase eine besondere Vertrauensstellung einnehmen.

In den letzten Jahren ist es dabei zu einer grundlegenden Veränderung im Dialog zwischen Arzt und Patienten gekommen. Das paternalistische Prinzip wurde von einem partnerschaftlichen Dialog gleichsam auf Augenhöhe - abgelöst.

\section{Lebensqualität und Nutzenbewertungen}

Lebensqualität. Das „Who ist Who“ aller Studien sollte in jedem Fall in einer Verbesserung der Lebensqualität der Menschen liegen, wobei meiner Meinung nach Qualität vor Quantität gehen sollte. Manchmal müssen wir den Patienten klar machen, dass die Zeit in ihrer Qualität etwas Unwiederbringliches ist - was heißt, dass es manchmal notwendig ist, den Patienten - in der Rezidivphase - Studien anzubieten, die v.a. der Verbesserung der Lebensqualität dienen, welche dabei auch als Bewertungsmaßstab jeder Thera-
Dieser Parameter dient nicht nur zur Beurteilung der direkten antitumoralen Effektivität eines Medikamentes, sondern trägt auch dazu bei, in Zeiten eingeschränkter Ressourcen den Schwellenwert für ein gewonnenes, qualitätskorrigiertes Lebensjahr nicht zu überschreiten. Laut „National Institute for Health and Clinical Excellence“ liegt dieser bei 40000 Euro.

Aus Sicht der Patienten kann der PFS zwar einen relevanten Gewinn bedeuten, doch um diesen so zu optimieren, dass sich die Menschenwürde nicht als ideologische Lehrformel erweist und der Wert des Menschen verloren geht, muss man in Zukunft sicherlich Diskussionen zur Ethik, Medizin und Ökonomie führen.

Eine Herausforderung sind die Wandlungsbilder in jedem Fall, ob sich der Stein der Weisen allerdings jemals findet, bleibt dahingestellt.

\section{Korrespondenzadresse}

PD Dr. Sonja Kickmaier

Gynäkologische Onkologie

AKH Wien

Währinger Gürtel 18-20

A-1090 Wien

E-Mail: Sonja.kickmaier@meduniwien.ac.at 


\section{Literatur}

1 Marth C. Overall Survival und Progression-free Survival in der Onkologie. GynAktiv 2014; 1: 20-21

2 Kickmaier S, Kölbl H. Klinische Studien - eine Herausforderung. Universum Innere Medizin 2014; 8: 64

3 Kickmaier S. Studien? Who is Who - von der Alchemie zum Biosimilar, wohin der Weg uns führt. Abstract vom Internationalen Kongress Menopause - Andropause - Antiaging; 2013
4 Kickmaier S. The power and glory of international studies - combining ethical and business objectives for the common good. Doctor Thesis AIU; 2011

5 Kickmaier S. Managing clinical studies - a critical reflection. Master Thesis AIU; 2010

6 http://seer.cancer.gov/csr/1975_2011; Stand: 15.05.2015

7 http://www.statistik.at/web_de/statistiken/gesundheit/europaeische_gesundheitsindikatoren_echi/index.html; Stand: 15.05.2015
8 http://www.ema.europa.eu/ema/index.jsp? curl=pages/regulation/landing/human_medicines_regulatory.jsp\&mid=; Stand: 15.05 .2015

9 http://www.fda.gov/Drugs/ScienceResearch/ default.htm; Stand: 15.05.2015

10 Arzneimittelgesetz. BGBI. Nr. 185/1983 zuletzt geändert durch BGBI. I Nr. 110/2012; § 2a 